\title{
Nonexistence of Positive Solutions to an Elliptic System and Blow-Up Rate for a Parabolic System
}

\author{
Z.Q. Ling \\ Institute of Mathematics and Information Science \\ Yulin Normal University \\ China
}

\begin{abstract}
We first get the conditions under which the elliptic system $-\Delta u_{i}=u_{i+1}^{p_{i}}, u_{s+1}:=u_{1}(i=1,2, \cdots, s)$ has no positive radially symmetric solutions. Then by using this nonexistence result, we establish blow-up estimates for semilinear reactiondiffusion system $u_{i t}=\Delta u_{i}+u_{i+1}^{p_{i}}, u_{s+1}:=u_{1}(i=1,2, \cdots, s)$ with null Dirichlet boundary conditions. The results of our paper with the those in Wang (Comp and Math with Appl, 44, $573-585,2002)$ are same, but our methods of proofs are entirely different, even easier than that used.
\end{abstract}

Keywords-elliptic system; parabolic system; blow-up; blow-up rate; nonexistence

\section{INTRODUCTION}

In the past decades, many physical phenomena have been formulated into local or nonlocal mathematical models of partial differential equation and studied by many authors, see [1-5] and the references therein. In particular, there exist many articles dealing with properties of positive solutions such as blow-up and blow-up rate to semilinear or degenerate parabolic equations. For example, Wang in [6] considered the following semilinear reaction-diffusion system

$\left(u_{i}\right)_{t}=\Delta u_{i}+u_{i+1}^{p_{i}}, u_{s+1}:=u_{1}, i=1,2, \cdots, s,(x, t) \in \Omega \times(0, T)$

And obtained the blow-up rate estimate

$$
c(T-t)^{-\lambda_{i}} \leq \max u_{i}(\cdot, t) \leq C(T-t)^{-\lambda_{i}},
$$

Where

$\lambda_{i}=\left(1+p_{i}+\sum_{l=i+1}^{i+s-2} p_{i} \cdots p_{l} /\left(p_{1} \cdots p_{s}-1\right), i=1,2, \cdots, s\right.$.

At the same time, the initial value problem and localized problem of (1) were studied by Pedersen [7] and Fila [8], respectively; they also obtained the same estimate (2) of blow-up rate.

By the motivations of the above cited works, in this paper, we will continue to study the blow-up rate of positive solutions for (1) with the following initial and boundary value conditions

$$
\begin{gathered}
u_{i}(x, 0)=u_{i 0}(x), \quad x \in \Omega, i=1,2, \cdots s, \\
u_{i}(x, t)=0, \quad(x, t) \in \partial \Omega \times(0, T), \quad i=1,2, \cdots s,
\end{gathered}
$$

Where $\quad \Omega \subseteq R^{N}, p_{1}, \cdots, p_{s} \geq 1, T>0 \quad$ and $u_{10}(x), \cdots, u_{s 0}(x)$ are continuous, nonnegative functions and vanish on $\partial \Omega$. By means of the non-existence result of the related elliptic system of (1) which obtained in next section, we establish the blow-up estimate (2). The results of this paper with those of [6] are same, but our methods of proofs are entirely different, even easier than that used. In addition, we always let $\Omega=B(0, R)=\left\{x \in R^{N} ;|x|<R\right\}, R>0$ denotes a open ball of $R^{N}$ centered at the origin of radius $R$ since we deal with positive radially symmetric solutions of system (1).

Before ending this section, we give the following lemma which will be used in our proofs. For convenience, we denote $p_{s+l}=p_{l}$ for all integers $l$ and

$$
A=\left(\begin{array}{cccccc}
1 & -p_{1} & 0 & \cdots & 0 & 0 \\
0 & 1 & -p_{2} & \cdots & 0 & 0 \\
\cdots & \cdots & \cdots & \cdots & \cdots & \cdots \\
0 & 0 & 0 & \cdots & 1 & -p_{s-1} \\
-p_{s} & 0 & 0 & \cdots & 0 & 1
\end{array}\right)_{(5)}
$$

Lemma 1 For the matrix $A$ which is defined by (5) and any constant $\varepsilon_{0}$, if det $A \neq 0$, then the following linear system

$$
A\left(y_{1}, y_{2}, \cdots, y_{s-1}, y_{s}\right)^{T}=\left(\varepsilon_{0}, \varepsilon_{0}, \cdots, \varepsilon_{0}, \varepsilon_{0}\right)^{T}
$$

has an unique solution $\left(y_{1}, y_{2}, \cdots, y_{s-1}, y_{s}\right)^{T}$ which is given by

$$
y_{i}=\frac{-\varepsilon_{0}\left(1+p_{i}+\sum_{j=1}^{s-2} p_{i} \cdots p_{i=j}\right)}{p_{1} p_{2} \cdots p_{s}-1}, y_{s+1}:=y_{1}, i=1,2, \cdots, s .
$$

\section{NONEXISTENCE FOR ELLIPTIC SYSTEM}

In this section, we investigate the following related elliptic system of (1)

$-\Delta u_{i}(x)=u_{i+1}^{p_{i}}(x), u_{s+1}:=u_{1}, i=1,2, \cdots, s, \quad x \in \Omega$ 
And consider the radially symmetric solutions of (7), i.e., suppose $u_{i}(x)=u_{i}(r)$ with $r=|x|, i=1,2, \cdots, s$. We have the following theorem.

Theorem 1 Assume that $N \geq 3$ and there exists $i \in\{1,2, \cdots, s\}$ such that $\delta_{i}>0$, or $\delta_{1}=\cdots=\delta_{s}=0$. Then (7) has no positive radially symmetric solution, where $\delta_{i}=2 \alpha_{i}-(N-2), i=1,2, \cdots, s$, here and next section, denote $\left(\alpha_{1}, \alpha_{2}, \cdots, \alpha_{s}\right)^{T}$ be a solution of linear system (6) with constant $\varepsilon_{0}=-1$.

To prove the Theorem 1, the system (7) can be rewritten in radial coordinates as

$$
\left(u_{i}\right)^{\prime \prime}+\frac{N-1}{r}\left(u_{i}\right)^{\prime}+u_{i+1}^{p_{i}}=0, u_{s+1}:=u_{1}, i=1,2, \cdots, s
$$

With $u_{i}^{\prime}(0)=0(i=1,2, \cdots, s)$. Secondly, similar to the Lemma 3.1 of [9], we have

Lemma 2 Let $\left(u_{1}, u_{2}, \cdots, u_{s}\right)$ be a positive, radially symmetric, decreasing solution of (8). Then for any $r>0$, we have

$$
\frac{r^{2}}{N} u_{i+1}^{p_{i}} \leq-r u_{i}^{\prime} \leq(N-2) u_{i}, \quad u_{s+1}:=u_{1}, \quad i=1,2, \cdots, s .
$$

Lemma 3 Suppose the conditions in Theorem 1 are satisfied. Let $\left(u_{1}, \cdots, u_{s}\right)$ be a positive and radially symmetric solution of (8). Then we have the following asymptotic estimates for $r>0$ :

$$
u_{i}(r) \leq(N(N-2))^{\alpha_{i}} r^{-2 \alpha_{i}}, \quad i=1,2, \cdots, s_{\text {(10) }}
$$

Proof of Theorem 1 Let $\left(u_{1}, u_{2}, \cdots, u_{s}\right)$ be a nontrivial positive and radially symmetric solution of (8). Next, we divide the proof into two cases.

Case i Without loss of generality, we assume $\delta_{1}>0$. By (9), $\left(r^{N-2} u_{1}\right)^{\prime} \geq 0$. Then we know that the function $r^{N-2} u_{1}$ is non-decreasing on $(0, \infty)$ and there exists a constant $c_{0}>0$ such that

$$
u_{1}(r) \geq c_{0} r^{-(N-2)}, \text { for } r>r_{0}>0
$$

Thus, (11) and (10) lead to a contradiction for $r$ sufficiently large since $\delta_{1}>0$.

Case ii Suppose that $\delta_{1}=0$ (the case $\delta_{i}=0, i=2, \cdots, s$, being similar). At first, by (10), we have

$$
r^{N-2} u_{1} \leq(N(N-2))^{\alpha_{1}} r^{(N-2)-2 \alpha_{1}}=(N(N-2))^{\alpha_{1}}
$$

Secondly, according to (8), we obtain

$$
r^{N-1}\left(-u_{1}^{\prime}(r)\right) \geq r^{N-1}\left(-u_{1}^{\prime}(r)\right)-r_{0}^{N-1}\left(-u_{1}^{\prime}\left(r_{0}\right)\right)=\int_{r_{0}}^{r} \sigma^{N-1} u_{2}^{p_{1}} d \sigma .
$$

At the same time, it follows from (9) that

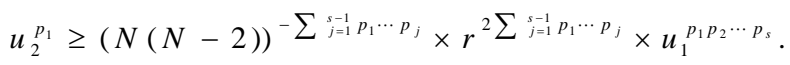

Together with (11), we obtain from (13) that

$$
r^{N-1}\left(-u_{1}^{\prime}\right) \geq c_{0}^{p_{1} \cdots p_{s}}(N(N-2))^{-\sum_{\substack{s-1 \\ j=1}}^{s} p_{1} \cdots p_{j}}\left(\ln \frac{r}{r_{0}}\right) .
$$

which will lead to a contradiction for $r$ sufficiently large. Thus, the proof of Theorem 1 is complete.

\section{BLOW-UP ESTIMATES FOR PARABOLIC SYSTEM}

Motivated by [1] and [2], we use the nonexistence result of the elliptic system obtained in section 2 to establish the blow-up estimate (2) for the reaction-diffusion system (1), (3) and (4). With the ideas in [10], we have the following lemma which is a comparison relationship between $u_{i}$ and $u_{i+1}$.

Lemma 4 If the exponents $p_{1}, p_{2}, \cdots, p_{s}$ of (1) satisfy $p_{i}\left(1+\sum_{j=1}^{s-2} p_{i+1} \cdots p_{i+j}\right) \geq(\leq) p_{i+1}\left(1+\sum_{j=2}^{s-1} p_{i+2} \cdots p_{i+j}\right), i=1, \cdots, s$.

Then the solution $\left(u_{1}, u_{2}, \cdots, u_{s}\right)$ of (1), (3) and (4) satisfies either

$u_{i+1}(x, t) \leq K_{i} u_{i}(x, t)^{\alpha_{i+1} / \alpha_{i}}, \alpha_{s+1}:=\alpha_{1}, u_{s+1}:=u_{1}, i=1,2, \cdots, s$.

or

$u_{i+1}(x, t) \geq L_{i} u_{i}(x, t)^{\alpha_{i+1} / \alpha_{i}}, \alpha_{s+1}:=\alpha_{1}, u_{s+1}:=u_{1}, i=1,2, \cdots, s$.

for all $(x, t) \in Q_{T} \backslash Q_{\eta}$, with some positive constants $K_{1}, \cdots, K_{s}, L_{1}, \cdots, L_{s}$ and $\eta \in(0, T)$.

Theorem 2 Let $\left(u_{1}, u_{2}, \cdots, u_{s}\right)$ be a classical solution of (1), (3) and (4), defined on $\Omega \times(0, T)$, with $(0, T)$ maximal time interval of existence, and $T<+\infty$. Assume that the following hypotheses are satisfied:

(i) $u_{1}(\cdot, t), \cdots, u_{s}(\cdot, t)$ are radially, decreasing, and symmetric functions of $r=|x|$;

(ii) $u_{i}(x, t), u_{i t}(x, t) \geq 0$ in $Q_{T}=\Omega \times(0, T), i=1,2, \cdots s$;

(iii) $u_{1 t}(x, t), \cdots, u_{s t}(x, t)$ achieve the maximum at $x=0$, for every $t \in(0, T)$;

(iv) $\delta_{i}>0$ or $\delta_{1}=\cdots=\delta_{s}=0$;

(v) $p_{i}\left(1+\sum_{j=1}^{s-2} p_{i+1} \cdots p_{i+j}\right) \geq p_{i+1}\left(1+\sum_{j=2}^{s-1} p_{i+2} \cdots p_{i+j}\right), i=1, \cdots, s$

Then there are constants $C_{1}, \cdots, C_{s}>0$ and $t_{1} \in(0, T)$ such that

$$
u_{i}(x, t) \leq u_{i}(0, t) \leq C_{i}(T-t)^{-\alpha_{i}}, i=1,2, \cdots, s
$$


for all $(x, t) \in Q_{T} \backslash Q_{t_{1}}$.

Remark 1 The conditions (i)-(iii) in Theorem 2 are reasonable if we impose appropriate assumptions on the initial data $u_{i 0}(x)$, for example, positivity, radial symmetry, and a suitable decreasing property with

$$
\Delta u_{i 0}+u_{(i+1) 0}^{p_{i}} \geq 0, u_{(s+1) 0}+=u_{10}, i=1,2, \cdots, s
$$

Proof of Theorem 2 Let $\left(\sigma_{1}, \sigma_{2}, \cdots, \sigma_{s}\right)$ be a solution of linear system (6) with constant $\varepsilon_{0}=-2$. Define for $t \in(0, T)$,

$$
\begin{gathered}
\rho_{1}(t)=u_{1}(0, t)^{1 / \sigma_{1}}, \rho_{2}(t)=u_{2}(0, t)^{1 / \sigma_{2}}, \cdots, \rho_{s}(t)=u_{s}(0, t)^{1 / \sigma_{s}} \\
\text { and } \rho(t)=\rho_{1}(t)+\rho_{2}(t)+\cdots+\rho_{s}(t) \text {. By putting } \\
w_{i}(r, t)=\frac{u_{i}(r / \rho(t), t)}{\rho(t)^{\sigma_{i}}}, i=1,2, \cdots, s
\end{gathered}
$$

and taking into account that $u_{i}(\cdot, t)$ achieve its maximum at $r=0$ since the assumption (i), we easily see that $w_{i}$ is bounded. On the other hand, it follows from the condition (iii) of Theorem 2 and Lemma 1 that

$$
0 \leq \Delta w_{i}(r, t)+w_{i+1}^{p_{i}}(r, t) \leq \frac{u_{i t}(0, t)}{\rho(t)^{\sigma_{i}+2}}, i=1,2, \cdots, s .
$$

By using the assumptions (i), (ii) and (18), we get

$$
0 \leq w_{i, r r}+\frac{N-1}{r} w_{i, r}+w_{i+1}^{p_{i}} \leq \sum_{j=1}^{s} \frac{u_{j t}(0, t)}{\rho(t)^{\sigma_{j}+2}}, i=1,2, \cdots, s
$$

for any $t \in(0, T)$ and $r \in(0[, R \rho(t))$. Multiplying the sum of (19) by $\left(w_{1}+\cdots+w_{s}\right)_{r}$ we have

$$
\frac{1}{2} \frac{d}{d r}\left(\sum_{i=1}^{s} w_{i}\right)_{r}^{2}+\frac{N-1}{r}\left(\sum_{i=1}^{s} w_{i}\right)_{r}^{2}+\left(\sum_{i=1}^{s} w_{i+1}^{p_{i}}\right)\left(\sum_{i=1}^{s} w_{i}\right) \leq 0
$$

which implies that

$$
\frac{1}{2} \frac{d}{d r}\left(\sum_{i=1}^{s} w_{i}\right)_{r}^{2}+\left(\sum_{i=1}^{s} w_{i+1}^{p_{i}}\right)\left(\sum_{i=1}^{s} w_{i}\right) \leq 0
$$

Integrating (20) on $(0, r)$ and together with $w_{i, r} \leq 0,0 \leq w_{i} \leq 1$, we obtain

$$
\frac{1}{2}\left(\sum_{i=1}^{s} w_{i}\right)_{r}^{2} \leq \sum_{i=1}^{s} \frac{1}{1+p_{i}}+s
$$

for any $t \in(0, T)$ and $r \in[0, R \rho(t))$. The inequality (21) implies the equicontinuity of $w_{i}, i=1,2, \cdots, s$.

Next, we claim that

$$
\liminf _{t \rightarrow T}\left(\frac{u_{1 t}(0, t)}{\rho(t)^{\sigma_{1}+2}}+\frac{u_{2 t}(0, t)}{\rho(t)^{\sigma_{2}+2}}+\cdots+\frac{u_{s t}(0, t)}{\rho(t)^{\sigma_{s}+2}}\right)>0
$$

Otherwise, there exists a sequence $\left\{t_{m}\right\} \subseteq(0, T)$ with $t_{m} \rightarrow T$ such that

$$
\liminf _{t \rightarrow T}\left(\frac{u_{1 t}(0, t)}{\rho(t)^{\sigma_{1}+2}}+\frac{u_{2 t}(0, t)}{\rho(t)^{\sigma_{2}+2}}+\cdots+\frac{u_{s t}(0, t)}{\rho(t)^{\sigma_{s}+2}}\right)=0
$$

By using the Ascoli-Alzela theorem, there exists a sequence (still denoted by $\left\{t_{m}\right\}$ ) such that

$$
w_{i}\left(\cdot, t_{m}\right) \rightarrow \bar{w}_{i}(\cdot), \quad m \rightarrow \infty(i=1,2, \cdots, s)
$$

uniformly on compact subsets of $[0,+\infty)$. Now in the sense of distributions

$$
\bar{w}_{i, r r}+\frac{N-1}{r} \bar{w}_{i, r}+\bar{w}_{i+1}^{p_{i}}=0, \quad i=1,2, \cdots, s
$$

The absolute continuity of $W_{i}$ implies that $\bar{w}_{i} \in C^{2}(0,+\infty)$ and, by local existence and uniqueness of the initial value problem for (23) and using the arguments in $[1,2]$, we conclude that $\bar{w}_{i}(r)>0$ on $(0,+\infty)$ with $\bar{w}_{i}^{\prime}(0)=0, i=1,2, \cdots, s$.

Now, in case $N=1$, we easily reach a contradiction by noting that, since (23) hold, then, $\bar{w}_{1}, \cdots, \bar{w}_{s}$ must be strictly concave on $(0,+\infty)$, which is impossible.

If $N=2$, we proceed as follows: from (23), we infer that $r \bar{w}_{1}^{\prime}, \cdots, r \bar{w}_{s}^{\prime}$ are strictly decreasing on $(0,+\infty)$. Hence there exist constants $M>0$ and $r_{0}>0$ such that $r \bar{w}_{i}^{\prime}<-M, r \in\left(r_{0},+\infty\right)$. This gives a contradiction when $r \rightarrow+\infty$.

If $N \geq 3$, we know from Theorem 1 that the elliptic system (23) has no positive solutions. Therefore, we conclude that (22) is true for all $N \geq 1$, that is, there exists a positive constant ${ }^{C}$ such that

$$
\liminf _{t \rightarrow T}\left(\frac{u_{1 t}(0, t)}{\rho(t)^{\sigma_{1}+2}}+\frac{u_{2 t}(0, t)}{\rho(t)^{\sigma_{2}+2}}+\cdots+\frac{u_{s t}(0, t)}{\rho(t)^{\sigma_{s}+2}}\right)=c>0
$$

It follows from (24) that there exists $t_{1} \in(0, T)$ such that $c \leq \frac{u_{1 t}(0, t)}{\rho(t)^{\sigma_{1}+2}}+\cdots+\frac{u_{s t}(0, t)}{\rho(t)^{\sigma_{s}+2}} \leq \frac{u_{1 t}(0, t)}{u_{1}(0, t)^{\left(\sigma_{1}+2\right) / \sigma_{1}}}+\cdots+\frac{u_{s t}(0, t)}{u_{s}(0, t)^{\left(\sigma_{s}+2\right) / \sigma_{s}}}$ holds for all $t \in\left(t_{1}, T\right)$. Integrating above inequality on $(t, \tau) \subseteq\left(t_{1}, T\right)$ and then letting $\tau \rightarrow T$, we have

$$
c(T-t) \leq \alpha_{1} u_{1}(0, t)^{-1 / \alpha_{1}}+\cdots+\alpha_{s} u_{s}(0, t)^{-1 / \alpha_{s}}
$$

Now, from Lemma 4, it follows that

$$
u_{i}(0, t)^{-1 / \alpha_{i}} \leq \mu_{i} u_{s}^{-1 / \alpha_{s}}, i=1,2, \cdots, s-1
$$

for some suitable constants $\mu_{i}>0$. Thus, (25) and (26) imply that 


$$
u_{s}(x, t) \leq u_{s}(0, t) \leq C_{s}(T-t)^{-\alpha_{s}}
$$

for all $(x, t) \in Q_{T} \backslash Q_{t_{1}}$. In order to obtain the analogous

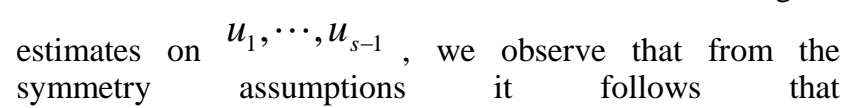
$\Delta u_{i} \leq 0, i=s-1, s-2, \cdots, 2,1$. Hence, from (1) and (27), we find that $u_{(s-1) t}(0, t) \leq u_{s}(0, t)^{p_{s-1}} \leq C(T-t)^{-p_{s-1} \alpha_{s}}=C(T-t)^{-1-\alpha_{s-1}}$.

Integrating the last inequality on $(0, t)$, it follows that

$$
u_{s-1}(x, t) \leq u_{s-1}(0, t) \leq C_{s-1}(T-t)^{-\alpha_{s-1}}
$$

for all $(x, t) \in Q_{T} \backslash Q_{t_{1}}$. Similarly, we can obtain the another estimates. Then the proof is complete.

Finally, we give lower bounds for the blow-up rates.

Theorem 3 Assume the conditions in Theorem 2 hold, then for $t \in(\eta, T)$, there are positive constant ${ }^{C_{1}}$ such that

$$
u_{i}(0, t) \geq c_{i}(T-t)^{-\alpha_{i}}, i=1,2, \cdots, s(28)
$$

Proof. For $t \in(0, T)$, since $\Delta u_{i} \leq 0$ at $x=0$ by the assumptions of Theorem 2, we see that $u_{i t}(0, t) \leq u_{i+1}(0, t)^{p_{i}}$. Together with Lemma 4, we obtain $u_{i t}(0, t) \leq u_{i+1}(0, t)^{p_{i}} \leq K_{i}^{p_{i}} u_{i}^{\left(p_{i} \alpha_{i}+1\right) / \alpha_{i}}=K_{i}^{p_{i}} u_{i}^{\left(1+\alpha_{1}\right) / \alpha_{i}}$

Integrating (29) over $(t, \tau) \subseteq(\eta, T)$ and then letting $\tau \rightarrow T$, we get the inequalities (28) hold for $t \in(\eta, T)$. The proof is complete.

Remark 2 Combining Theorems 2 and 3, we conclude that the blow-up rates of radial positive solutions of the reaction-diffusion system (1), (3) and (4) under the conditions of the theorems are $u_{i}(0, t)=O\left((T-t)^{-\alpha_{i}}\right), i=1,2, \cdots, s$, as $t$ tends to $T$.

\section{ACKNOWLEDGEMENTS}

This work is supported in part by NNSF of China (11461076) and in part by Universities and colleges research foundation of Guangxi (ZD2014106).

\section{REFERENCES}

[1] Weissler, F.B., An $L^{\infty}$ blow-up estimate for a nonlinear heat equation. Communications on Pure and Applied Mathematics, 38(3), pp. 291295, 1985.

[2] Caristi, G. \& Mitidieri, E., Blow-up estimates of positive solutions of a parabolic system. Journal of Differential Equations, 113(2), pp. 265-271, 1994.

[3] Z.Q. Ling \& Z.J. Wang, Blow-up problems for a compressible reactive gas model. Boundary Value Problems 2012, 2012:101.

[4] Z.Q Wu \& J.N. Zhao \& J.X. Yin \& H.L. Li, Nonlinear diffusion Equations. World Scientific, River Edge NJ, 2001.

[5] S.N. Zheng \& B.C. Liu \& F.J. Li, Blow-up rate estimates for a doubly coupled reaction-diffusion system. Journal of Mathematics Analysis and Applications, 312, pp.576-595, 2005.
[6] M.X Wang, Blow-up rate for a semilinear reaction diffusion system. Computers \& Mathematics with Applications, 44, pp. 573-585, 2002.

[7] Pedersen, M. \& Z.G. Lin, Coupled diffusion systems with localized nonlinear reactions. Computers \& Mathematics with Applications, 42, pp. 807-816, 2001.

[8] Fila, M. \& Quittner, P., The blow-up rate for a semilinear parabolic system. Journal of Mathematics Analysis and Applications, 238, pp. 468-476, 1999.

[9] Mitidieri, E., A Rellich type identity and applications. Communications in Partial Differential Equations, 18, pp. 125-171, 1993.

[10] A Friedman \& Y Giga. A single point blow-up for solutions of semilinear parabolic systems. Journal of the Faculty of Science, University of Tokyo, Sect IA Math, 34, 65-79, 1987. 